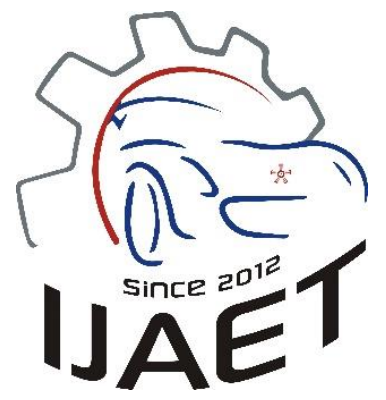

e-ISSN: 2146 - 9067

International Journal of Automotive

Engineering and Technologies

journal homepage: http://ijaet.academicpaper.org

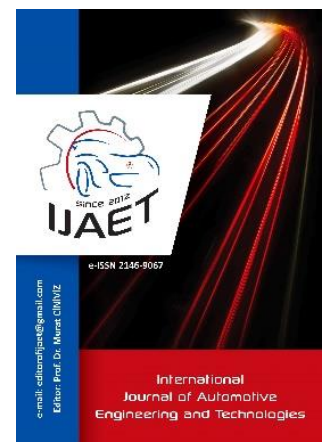

Original Research Article

\title{
Numerical analysis of elastomer buffer embedded in the suspension of automobile for vibration damping improvement
}

hosted by

Turkish

\author{
Ahmad Partovi Meran ${ }^{1, *}$ \\ ${ }^{1}$ Department of Mechatronics Engineering, Faculty of Engineering and Architecture, N. Erbakan University
}

\begin{abstract}
ARTICLE INFO
* Corresponding author

ameran@konya.edu.tr

Received: February 09, 2018

Accepted: March 21, 2018

Published by Editorial Board Members of IJAET

(C) This article is distributed by Turk Journal Park System under the CC 4.0 terms and conditions.
\end{abstract}

\begin{abstract}
Elastomers, due to their excellent damping and energy absorption characteristics and low cost are used extensively in automobile industry to isolate the structures from vibration and shock loads. In this study, it was aimed to analyze the damping performance of an elastomer buffer embedded in the suspension of an automobile. To reach to this aim, vibration simulation of an automobile suspension model was conducted by using a nonlinear explicit finite element code, Abaqus. In order to simulate the damping behavior of elastomer buffer, the hyperelastic and linear viscoelastic material models were used together. The numerical model was validated with results of exact solution method in terms of transmissibility ratio and phase shift in a wide range of input excitation frequencies. Good agreement was observed between the exact solution and finite element results, which indicated that finite element model was sufficiently accurate. To examine the damping performance of the buffer, the displacement time history curves were extracted for suspension with and without buffer under the sinusoidal base excitations. The vibrating motions of suspension for both conditions were compared. The comparison results prove that the elastomer buffer is effective in improvement of damping performance of suspension. It reduces the amplitude of vibration and oscillation time of sprung mass remarkable in excitation frequencies around and over the natural frequency of the system.
\end{abstract}

Keywords: Elastomer, Viscoelasticity, Suspension, Buffer, Viscoelastic damping

\section{Introduction}

Elastomers, due to their excellent damping and energy absorption characteristics, moldability, variable stiffness and low cost are used extensively in automobile industry to isolate the structures from vibration and shock loads. Many polymeric materials due to having long chain molecules represent viscoelastic behavior [1]. Viscoelastic materials exhibit the composed characteristics of an elastic solid and a viscous liquid which under mechanical loading represent three common characteristics; creep, stress relaxation and hysteresis. Creep is the continuous deformation of a material under constant load. Stress relaxation is the progressive decrease of stress when a material subjected to a constant strain. Hysteresis refers to a difference between loading and unloading paths in the stress-strain diagram of a material. The area enclosed by the paths indicates the energy dissipated as heat when the material deforms and recover [2].

The response of a viscoelastic material to an applied load depends on the time and rate of loading. Consequently, the stress-strain diagram 
for a viscoelastic material is a function of time and strain rate. In order to characterize the viscoelastic behavior of materials, researchers conducted theoretical, experimental and numerical studies [3-7]. For instance, Busfield et al. investigated the dynamic storage and loss moduli of rubber samples by free oscillation technique [8]. Boltzmann proposed the superposition principle composing the basic of linear viscoelasticity theory which applicable for small strain and small strain-rate regime. Linear viscoelastic material follows the small strain regime behavior and the stress-strain relation can be defined by linear differential equations with constant coefficients [9]. The characteristic response of a linear viscoelastic material such as stress relaxation and creep can be well approximated by Maxwell and Voigt model, respectively. Sometimes to predict the linear viscoelastic behavior of a material, several Maxwell elements in parallel to a single spring known as Prony-series are used [1].

In literature there are a lot of reported studies concerning application of viscoelastic material for passive vibration damping in automobile structures. The reported studies related to viscoelastic damping treatments can be summarized in three ways: free-layer damping, sandwich-layer and tuned viscoelastic damping [10]. In industrial applications elastomeric isolators, rubbers and viscous isolators are widely being employed. Mallik et al. [11] experimentally investigated the restoring and damping characteristics of elastomeric isolators. Richards and Singh characterized the nonlinear parameters of rubber isolators in multi-degreeof-freedom systems [12]. Chandra et al. showed that the stiffness and damping characteristics of viscoelastic dampers are non-linear [13]. Shaska et al. compared the characteristics of a nonlinear viscoelastic isolator with a linear vibration isolator. The results showed that the stiffness and damping of viscoelastic isolator were dependent on frequency and temperature. Furthermore, it was observed that the nonlinear isolator improved the transmissibility [14]. Sjoberg and Kari experimentally investigated the effects of nonlinear excitation on the dynamic stiffness and damping of a rubber isolator. It was found that the stiffness was highly nonlinear and damping depended on the amplitude of single harmonic excitation [15].
Banic et al. numerically studied the heat generation of a rubber damper under cyclic loading and to predict the hysteresis pattern and heat generation utilized the visco-plastic constitutive model established by BergstromBoyce [16]. Huang et al. numerically and experimentally analyzed the dynamic characteristics of a viscoelastic damping isolator under impact loading [17].

Since, at large strain the linear viscoelasticity theory under-predicts the size of the hysteresis loop, the error in the dissipated energy prediction is not acceptable [18]. For this reason, in hysteresis analysis the hyperelasticity and linear viscoelasticity are used together. The stress response of the hyper-viscoelastic model consists of a nonlinear elastic part which is instantaneous and a viscous part which is extended over time. Also, there are some studies analyzing viscoelastic material by a lumped spring-damping system. For instance, Luo et al. [19-20] conducted fatigue analysis on the suspension systems of a rail vehicle by replacing rubber with spring and damping elements and found reasonable results. Grassie [21] calculated the dynamic stiffness of a rubber pad by using a lumped spring-damping system and obtained a good agreement between experimental results and dynamic model ones. Also, in literature there are reported studies concerning the effect of road roughness and vehicle velocity on the vibration performance and fatigue life of automobile suspension system [22-24]. In these studies, a quarter car model used to study the suspension system performance under the random vibration with variable amplitude loading.

In literature there is no study focused on the analysis of an elastomeric buffer embedded on a coil spring. Motivated by this fact, this study was initiated to investigate numerically the effect of elastomer buffer on the vibration behavior of automobile suspension system. The commercial finite element (FE) software Abaqus was employed to conduct the numerical analysis of a typical coil spring which belongs to a light vehicle. One quarter mass of a vehicle with four persons and fuel was added to the head of the spring and under various base motions the vibration of the sprung mass was observed. The numerical model was validated with results of 
the exact solution method in terms of transmissibility ratio and phase shift in a wide range of input excitation frequencies. Good agreement was observed between the exact solution and finite element results, which indicate that finite element model is sufficiently accurate. Then, an elastomer buffer which was fabricated from thermoplastic was mounted on the coil spring. The mechanical behavior of thermoplastics is highly nonlinear and sensitive to loading rate. To simulate the behavior of buffer under shock loading and vibration, nonlinear viscoelasticity and hyperelastic material models were utilized. Finally, under the same base motion inputs, the vibration of the sprung mass was measured. The compared numerical results proved the effectiveness of the elastomer buffer in improvement of damping performance of suspension. It reduced the amplitude of vibration and oscillation time of sprung mass remarkable in excitation frequencies around and over the natural frequency of the system.

\section{Viscoelastic damping}

In simple terms, damping refers to the extraction of mechanical energy from a vibrating structure and conversion into heat. Damping of viscoelastic material is measured as a loss factor $(\eta)$. Since, mechanical properties of the viscoelastic dampers are highly sensitive to the amplitude and frequency of the excitation and ambient temperature, the loss factor is measured at the range of frequencies and temperatures. At lower frequencies the material has time to respond to vibration and the viscous characteristic dominates the material response [2]. But at high frequencies the material has no enough time to respond. So the elastic characteristic dominates the material response. There is an optimal frequency range where loss factor peaks. To represent the elastic and viscous properties of viscoelastic material, the complex shear modulus is used as presented in Eq.1. The complex shear modulus includes the storage $\left(G^{\prime}\right)$ and loss $\left(G^{\prime \prime}\right)$ shear moduli. The ratio of the loss to storage modulus is the loss factor, $\eta$, or so-called tangent delta $(\tan \delta)$ Eq.2. The loss angle is the phase angle between stress and strain during sinusoidal deformation in time [1]. To describe the material, the tangent delta along with storage modulus $\left(\mathrm{G}^{\prime}\right)$ are used. The loss angle or the loss tangent is a measure of damping in a linear viscoelastic material.

$G^{*}=\mathrm{G}^{\prime}+\mathrm{i} \mathrm{G}^{\prime \prime}$

$\eta=\frac{\mathrm{G}^{\prime \prime}}{\mathrm{G}^{\prime}}=\tan \delta$

Since, under high strain loading viscoelastic material exhibits nonlinear behavior, to calculate the shear moduli which represent the mechanical properties of damper a linearization technique can be used. Viscoelastic dampers under large strain can be characterized by using small strain modulus data. Experiments have shown that vibration frequency or the rate of loading has significant effect on the damping and dynamic modulus of viscoelastic materials [2].

\section{Finite element simulation}

In order to analyze the effect of embedded elastomeric buffer on the vibration behavior of suspension, simplified one quarter car's suspension model is considered as shown in Fig 1. A complete quarter car's suspension model includes sprung mass, unsprung mass, spring, damper, tire spring and tire damper [22]. In this study, in order to conduct a comparison between suspension systems including embedded elastomeric buffer and systems without buffer and due to limited CPU capacity a simplified model is used. A simplified one quarter car's suspension model can be considered as a system attached to the base via a coil spring and a viscous damper. Coil springs, also known as helical springs, are widely used on the suspension systems of light vehicles to store energy and keep the contact of traveling wheel with ground. They are fabricated from hardened and tempered steel, wound in a spiral formation. The damper reduces the magnitude of the motion transmitted from the ground to the automobile.

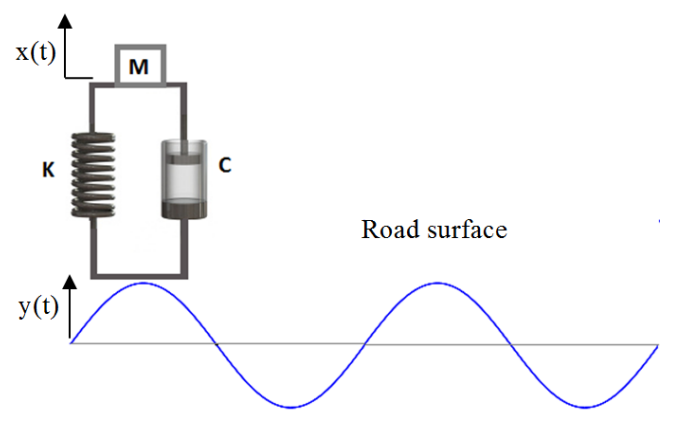

Figure 1. Dynamic model 
Abaqus/Standard and Explicit software was used to study the effect of elastomer buffer on the behavior of suspension system. The analysis was conducted for suspension without buffer and with buffer individually. First, the suspension model without buffer was modeled individually and validated by exact solution method. Then, on the validated model the buffer embedded on the coil spring analysis was conducted. The isotropic elastic-plastic material model with $1445 \mathrm{MPa}$ yield strength, $209 \mathrm{GPa}$ elasticity modulus, 0.29 Poission's ratio and $1545 \mathrm{MPa}$ ultimate strength was assigned to the coil spring. The solid 3D stress element type of C3D10M (a 10-node modified quadratic tetrahedron) was assigned to the coil spring. To ensure the convergence of numerical results, various mesh sizes were utilized and finally 15507 elements were generated on the coil spring model. One-quarter mass of a car about $250 \mathrm{~kg}$ was added to the top of the spring. The nodes at the bottom of the spring were constricted at all directions. In order to simulate the viscous damper of suspension, dashpots element known as DASHPOTA with 2 Ns/mm dashpot coefficient was utilized. This element is used to model relative velocity-dependent force or torsional resistance. It can also provide viscous energy dissipation mechanisms. This element is axial dashpot between two nodes, whose line of action is the line joining the two nodes. It introduces a damping force between two degrees of freedom without introducing any stiffness between these degrees of freedom and without introducing any mass at the nodes. This can cause a reduction in the stable time increment. In Abaqus it is available to define linear dashpot behavior by specifying a constant dashpot coefficient (force per relative velocity). It is also possible to define nonlinear dashpot behavior by giving pairs of force-relative velocity values. Static analysis was conducted to obtain equilibrium position of coil spring under the gravity force. Then, sinusoidal displacement excitation input with various amplitudes and frequency spectrum were assigned to the base of the suspension. Explicit dynamic analysis was conducted with sine harmonic displacement excitations for a frequency range as $f=$ $0.25,0.5,1,2,3,4,5,10$ and $20 \mathrm{~Hz}$ and $50 \mathrm{~mm}$ amplitude. Quarter of automobile travelling over a rough road is simply modeled as a single degree of freedom as shown in Fig. 1. The automobile attached to the base via a spring and a damper. The base is excited by the displacement input function $y(t)$. Based on Fig. 1 , the equation of motion of the sprung mass $(M)$ can be written as;

$M \ddot{x}+k(x-y)+c(\dot{x}-\dot{y})=0$

The road profile is idealized by a sine harmonic, $y(t)=Y \sin \left(\omega_{f} t\right)$. The steady state solution gives the displacement transmissibility ratio as Eq.4. The phase shift can be determined by Eq. 5.

$\frac{X}{Y}=\sqrt{\frac{1+(2 \zeta r)^{2}}{\left(1+r^{2}\right)^{2}+(2 \zeta r)^{2}}}$

where, damping ratio, $\zeta=\frac{c}{2 \sqrt{\mathrm{km}}}$ and natural frequency of system is $\omega_{n}^{2}=\frac{k}{m} \cdot r$ is frequency ratio, $r=\frac{\omega_{f}}{\omega_{n}}, \omega_{f}$ is excitation frequency. $k$ and $c$ are the spring rate constant and coefficient of damper, respectively.

$\varnothing=\tan ^{-1}\left(\frac{2 \zeta r^{3}}{1-r^{2}+(2 \zeta r)^{2}}\right)$

The spring rate constant of a coil spring can be calculated by following equation.

$k=\frac{G d^{4}}{8 n D^{3}}$

where $\mathrm{G}$, is the shear modulus of spring material, $G=\frac{E}{2(1+v)}$ where $d, n$ and $D$ are wire diameter, number of active coils and coil mean diameter, respectively. Under tension or compression, the material (wire) of a coil spring undergoes torsion. The spring characteristics therefore depend on the shear modulus, not Young's Modulus. The numerical model was validated with results of exact solution method in terms of transmissibility ratio and phase shift in a wide range of input excitation frequencies. As shown in Figures 2 and 3, good agreement was observed between the exact solution and finite element results, which indicate that finite element model is sufficiently accurate.

After validation of FE model, the elastomer buffer model was established in Abaqus. Figure 4 shows a typical elastomer buffer which is commercially known as power cushion buffer. The CAD model and meshed model are shown in Figure 5 (a) and (b). 


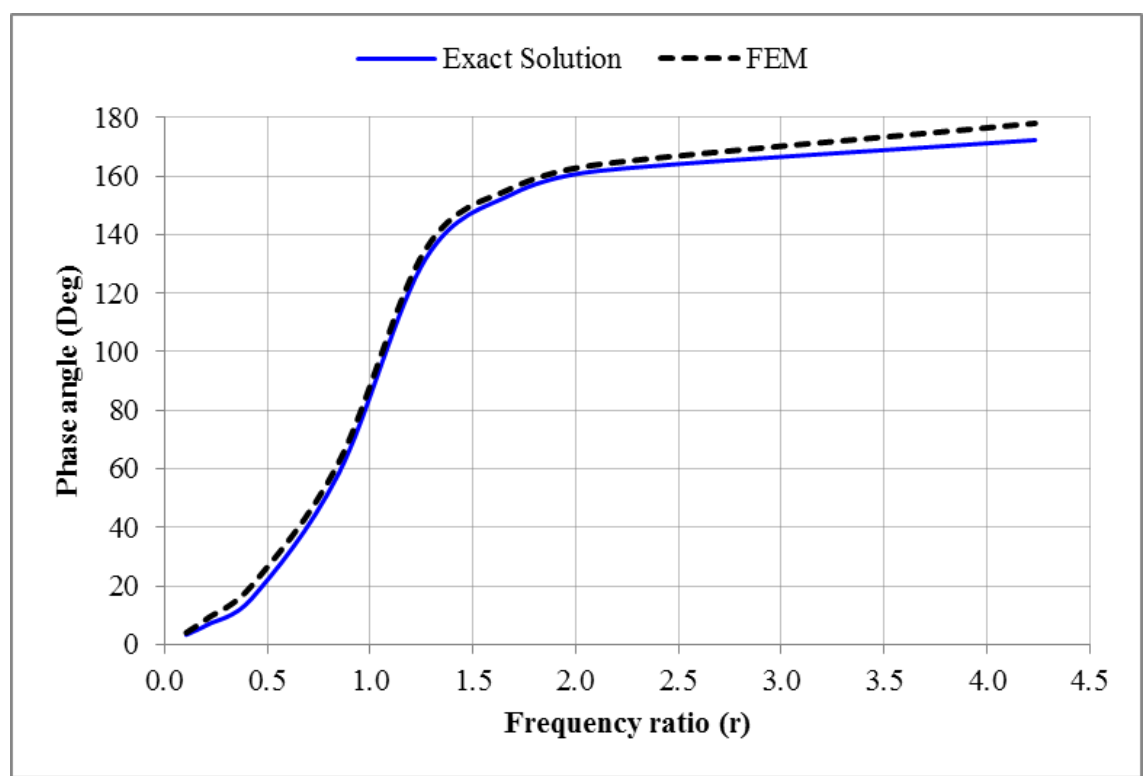

Figure 2. Phase angle vs. frequency ratio(r)

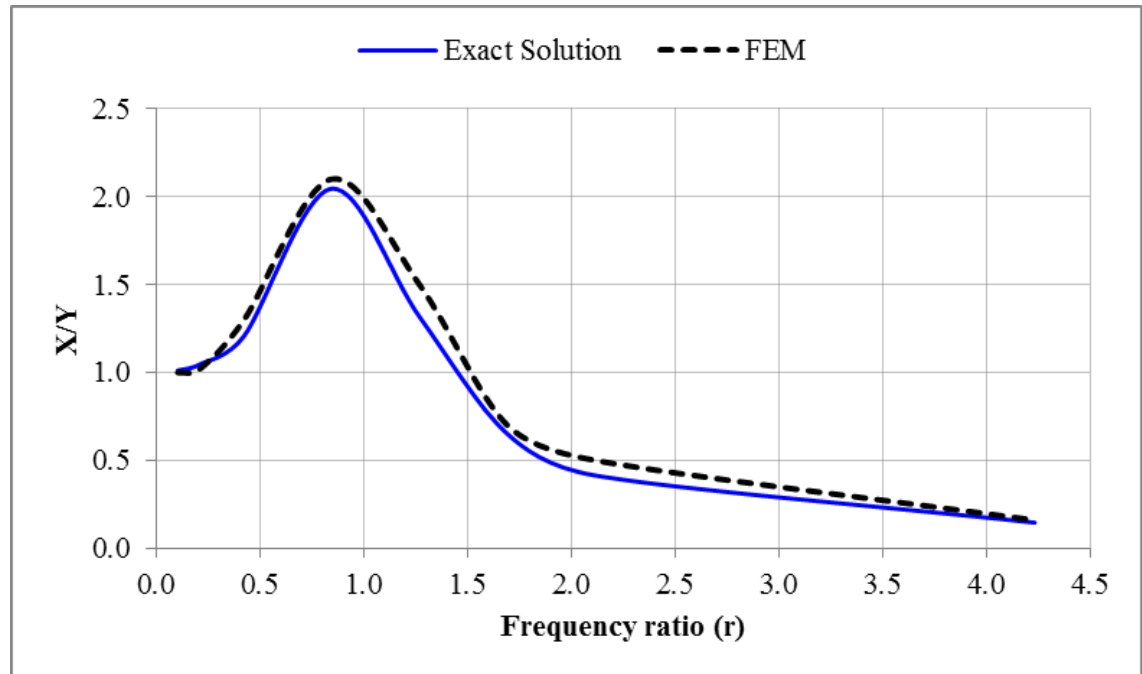

Figure 3. Displacement transmissibility ratio (TR) vs. frequency ratio

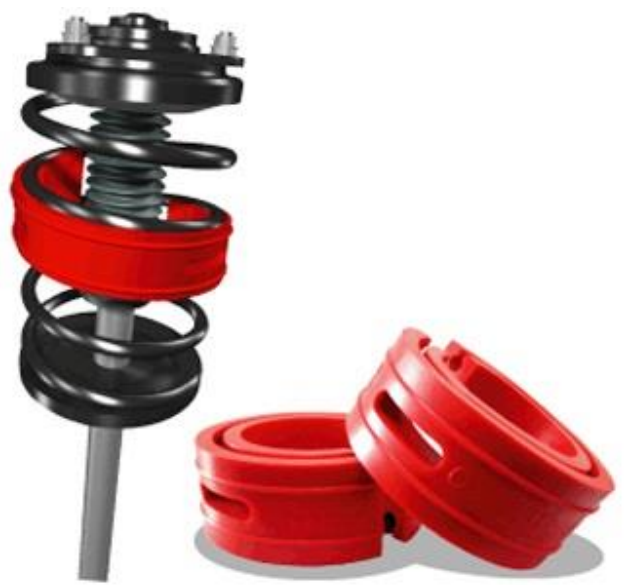

Figure 4. Commercially available elastomer buffer [25]

In order to capture the loading rate effects on elastomer buffer, viscoelastic material and to simulate the nonlinear elasticity of elastomer buffer a hyperelastic material model are used in Abaqus. To simulate the rubber like material in abaqus, it is essential to calibrate the model. The hyperelastic and viscoelastic material models parameters are calibrated separately by Abaqus. The hyperelastic model is calibrated with quasistatic tension and volumetric test data [26]. 

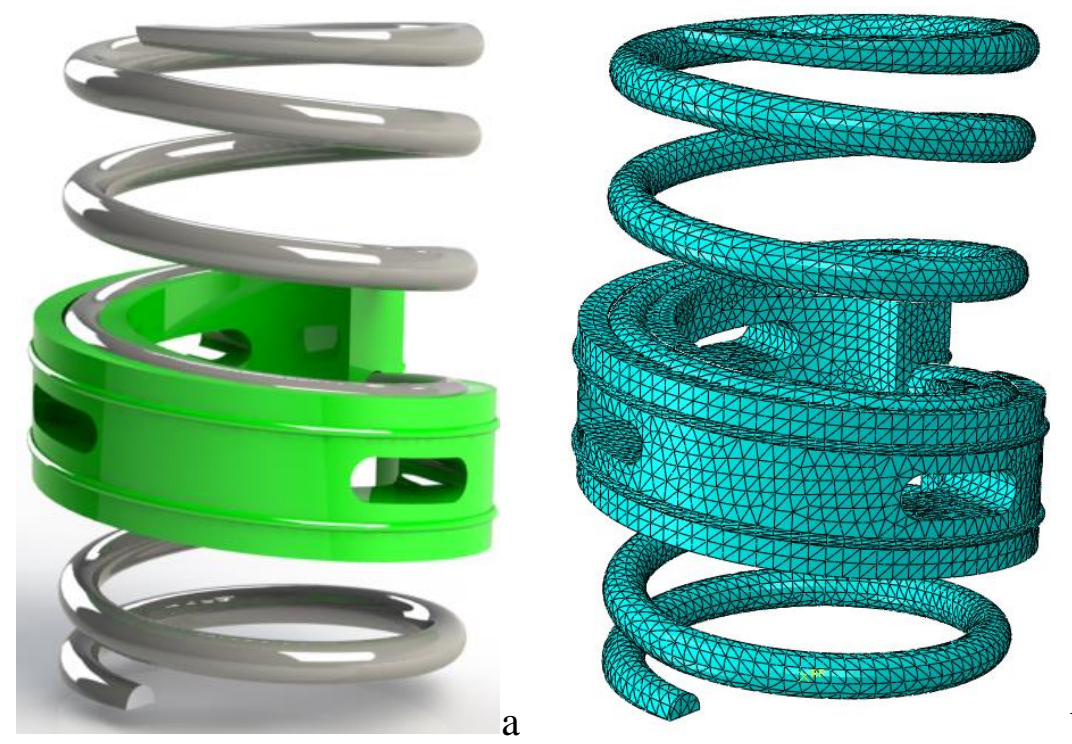

b

Figure 5. a) CAD model of coil spring with mounted elastomer buffer and b) Full-scale FE model

Most elastomers in comparison to their shear flexibility have very small compressibility compared. In all hyperelastic models, it is assumed that the material behaves isotropic throughout the deformation history. Hence, the strain energy potential can be formulated as a function of the strain invariants. Hyperelastic materials are described in terms of a strain energy potential which defines the strain energy stored in the material per unit of reference volume as a function of the strain at the point in the material. There are several forms of strain energy potentials available in Abaqus to model approximately incompressible isotropic elastomers. When only one set of test data (uniaxial) is available the Marlow form is recommended. In this case a strain energy potential is constructed that will reproduce the test data exactly and that will have reasonable behavior in other deformation modes. The mechanical response of a material is defined by choosing a strain energy potential to fit the particular material. The elastic response of viscoelastic materials and hysteretic materials can be specified by defining either the instantaneous response or the long-term response of such materials. The most straight forward generalization of Hooke's law to large displacements and large deformations is hyperelasticity [26].

The combined hyperelastic and linear viscoelastic material model was used to simulate the elastomer buffer material. Abaqus/CAE provides some hyperelastic material models. Here, Yeoh hyperelastic model was assigned. In this model, the strain energy density function is described as follows:

$W=\sum_{i=1}^{N} C_{i 0}\left(I_{1}-3\right)^{i}+\sum_{k=1}^{N} \frac{1}{D}(J-3)^{2 k}$

where $\mathrm{C}_{\mathrm{i} 0}$ are material constants, $\mathrm{J}$ is the determinant of the strain gradient tensor F. D is a material constant which controls compressibility. The viscoelastic prony series parameters and hyperelastic parameters were taken from Ref. no. 26. are listed in Table 1. The solid 3D stress element type of C3D10M (a 10node modified quadratic tetrahedron) was assigned to the elastomer buffer. To ensure the convergence of numerical results, various mesh sizes were utilized and finally 22458 elements were generated on the buffer model as shown in Fig 5 (b).

Table 1. Viscoelastic prony series parameters

and hyperelastic parameters [27]
\begin{tabular}{|c|c|c|c|}
\hline$C_{10}$ & 0.1524 & tau $_{1}$ & 1.354 \\
\hline$C_{20}$ & -0.0079 & $g_{2}$ & 0.06761 \\
\hline$C_{30}$ & 0.0023 & tau $_{2}$ & 49.5647 \\
\hline$D_{1}$ & 0.1230 & $g_{3}$ & 0.0529 \\
\hline$g_{1}$ & 0.0560 & tau $_{3}$ & 986.9 \\
\hline
\end{tabular}

\section{Results and Discussion}

In this section, the results of FE simulation of suspension under the base excitation are presented. In order to assess the effect of viscoelastic buffer on the vibration damping of 
car's suspension, displacement vs. time curves of sprung mass were extracted for both conditions; with buffer and without buffer. Figures 6 (a) and (b) show the Von Mises stress distribution on the suspension. A shown in Fig.
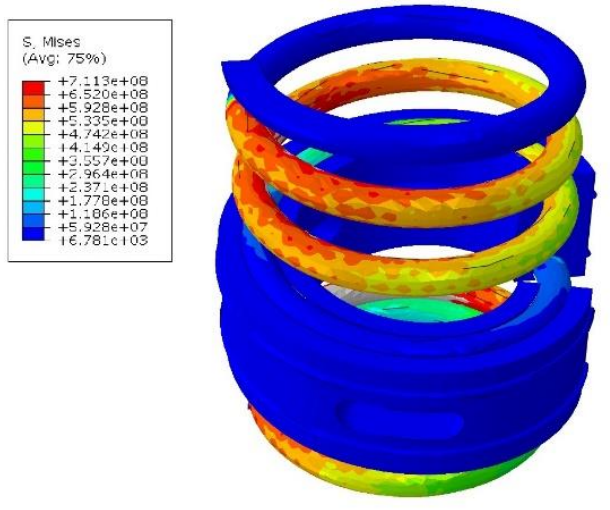

a
6 a), the level of stress on the spring is higher than the buffer. In order to visualize the stress distribution on the buffer the maximum stress limit was modified in Abaqus visualization module.
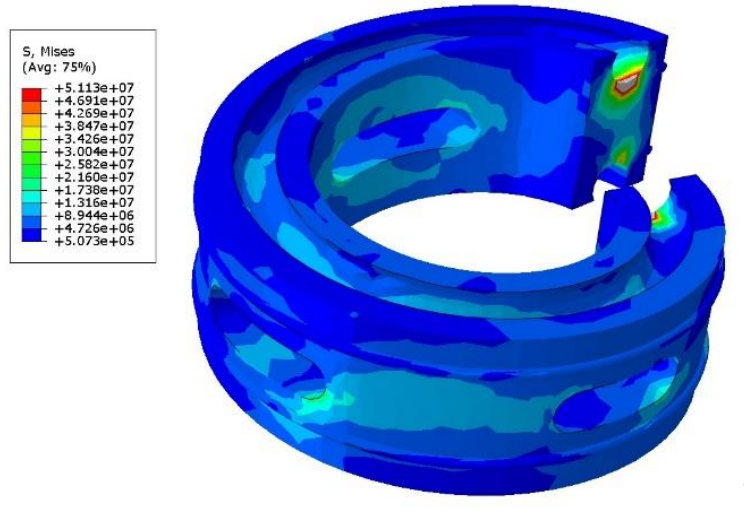

b

Figure 6. a) Von Mises stress distribution on coil spring and b) Von Mises stress distribution on buffer

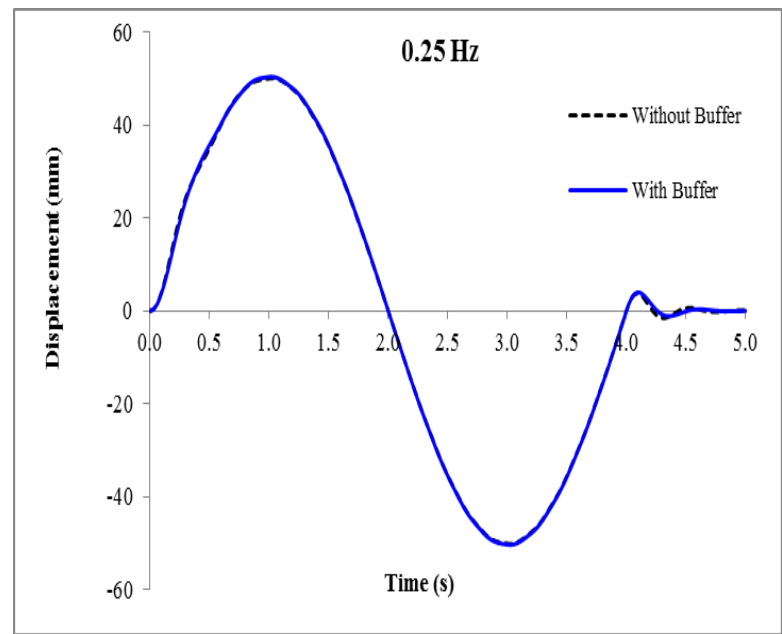

Figure 7. Displacement vs. time of the sprung mass for $0.25 \mathrm{~Hz}$

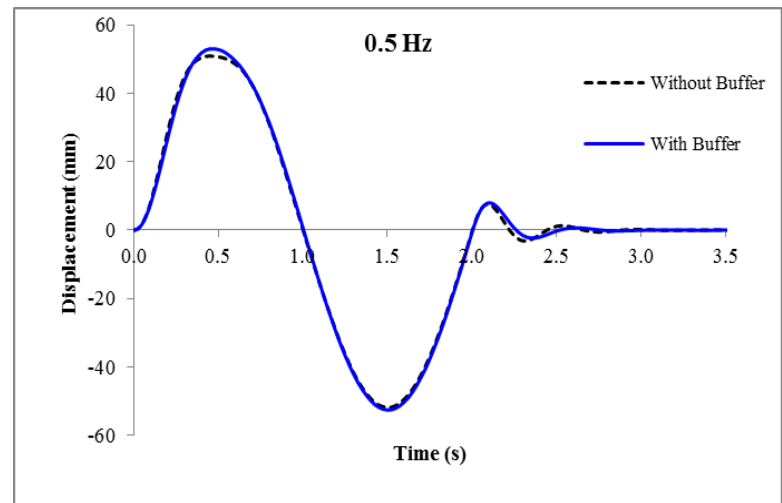

Figure 8. Displacement vs. time of the sprung mass for $0.5 \mathrm{~Hz}$

Figures 7 to 15 illustrate the displacement of sprung mass respect to the time for system with buffer and without buffer. In order to analyze the vibration motion of system, damping ratio is one of the important parameters. The damping ratio of system $\zeta=\frac{c}{2 \sqrt{\mathrm{km}}}$ with considering $k=$ $55(\mathrm{~N} / \mathrm{mm}), c=2(\mathrm{Ns} / \mathrm{mm})$ and $m=250 \mathrm{~kg}$ can be obtained $\zeta=0.002$. In this case the square root of $\zeta^{2}-1$ is an imaginary number. This indicates that the damping of system is insufficient to prevent vibration and the motion is oscillatory. As shown in Figures 7 to 9, it is observed that in frequencies under $1 \mathrm{~Hz}$, the base motion transferred to the sprung mass directly and there is not remarkable distinction on the vibrating motion behavior of system with and without buffer in frequencies under $1 \mathrm{~Hz}$. In other words, in frequencies lower than the natural frequency of system the elastomeric buffer is not effective in vibration damping.

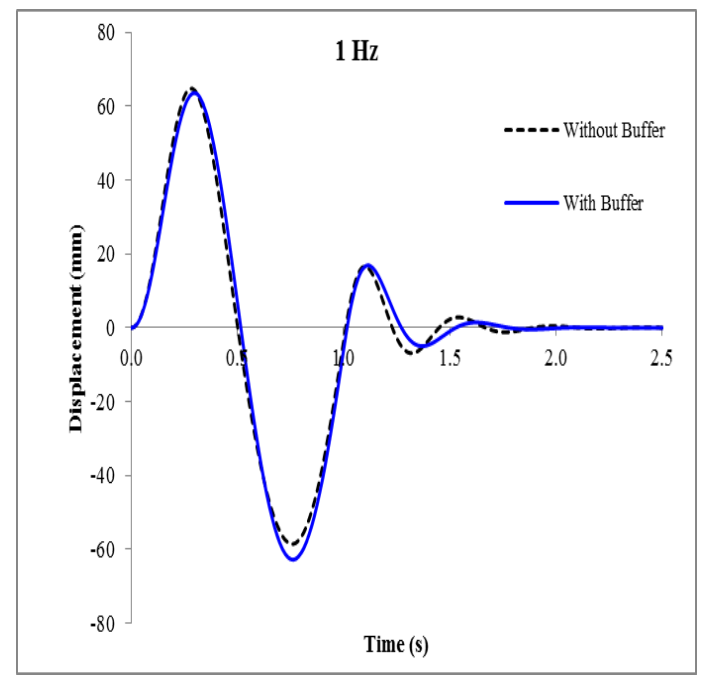

Figure 9. Displacement vs. time of the sprung mass for $1 \mathrm{~Hz}$ 
By considering Figure 3 it comes out that the damped natural frequency of system without buffer is between 2 and $3 \mathrm{~Hz}$. The relation between the damped natural frequency and undamped natural frequency is expressed by Eq. 8 With attention to that the undamped natural frequency of system equals to $\omega_{n}=2.36 \mathrm{~Hz}$ by substituting to Eq. 8 the damped natural frequency of system can be obtained as $\omega_{d}=$ $2.3599 \mathrm{~Hz}$. The frequency ranges between 2 and $3 \mathrm{~Hz}$ are resonance area of system. As shown in Fig. 10 and 11 the output amplitude of the system is higher than the amplitude of input harmonic signal. Also it can be observed that amplitude of system with buffer is lower than the system without buffer. It is especially distinctive after the second cycle. In system without buffer the oscillating motion is damped after four cycles in $2 \mathrm{~Hz}$. But is system with buffer the vibrating motion is damped after three cycles. The same manner can be observed in the oscillation with input frequency of $3 \mathrm{~Hz}$.

$\omega_{d}=\omega_{n} \sqrt{1-\zeta^{2}}$

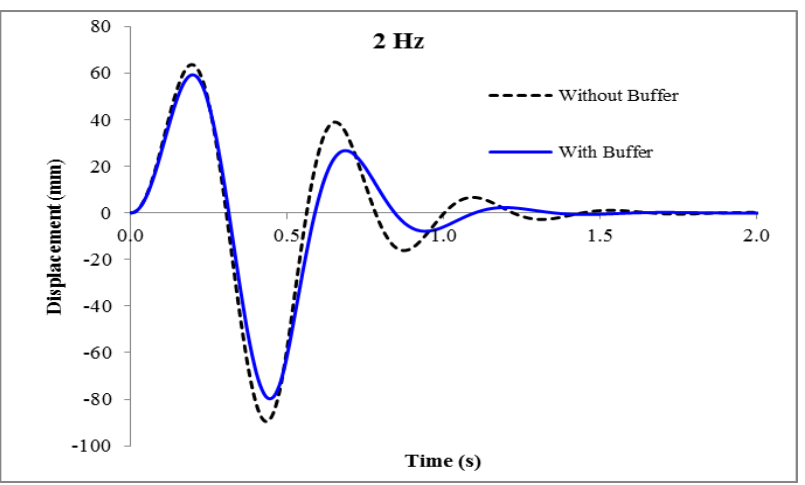

Figure 10. Displacement vs. time of the sprung mass for $2 \mathrm{~Hz}$

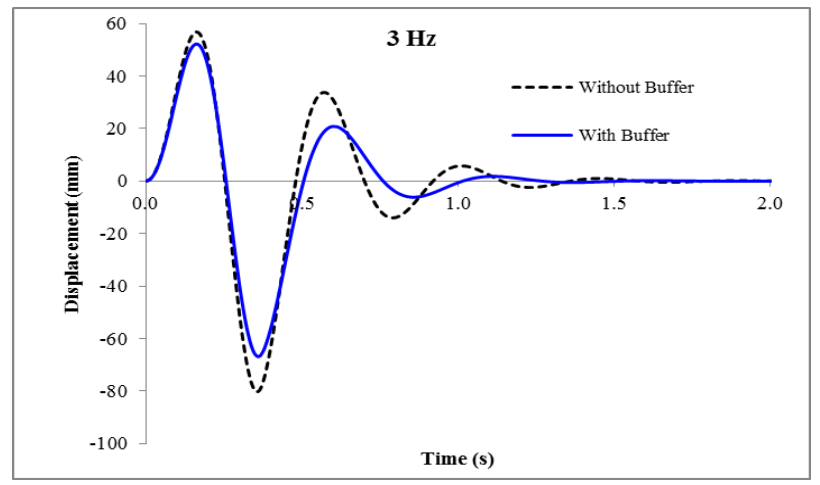

Figure 11. Displacement vs. time of the sprung mass for $3 \mathrm{~Hz}$

Figures 12 and 13 show the displacements of sprung mass respect to time in frequencies 4 and $5 \mathrm{~Hz}$. In frequencies around two times of natural frequency, it is observed that the amplitude of sprung mass is lower than the input one. Also by increasing the input frequency the output amplitude is reduced. The effectiveness of buffer in reduction of vibration amplitude and oscillation time is noticeable. The oscillation motions of systems with and without buffer are damped after two and three cycles, respectively.

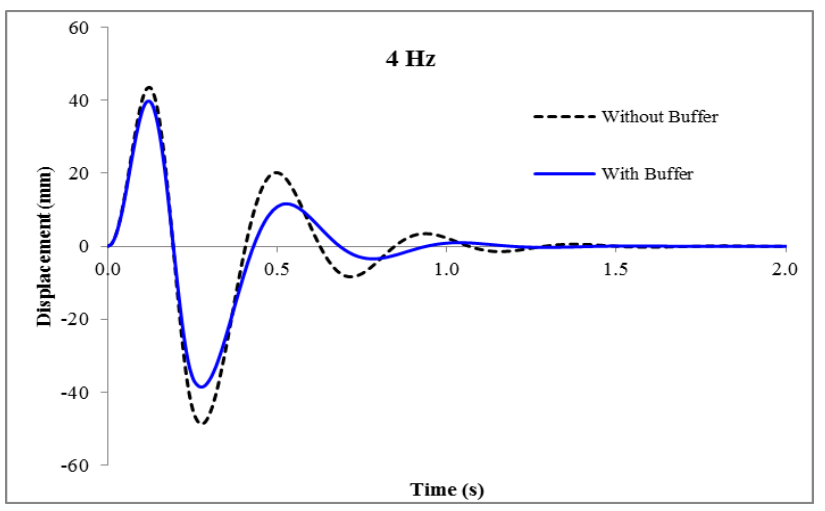

Figure 12. Displacement vs. time of the sprung mass for $4 \mathrm{~Hz}$

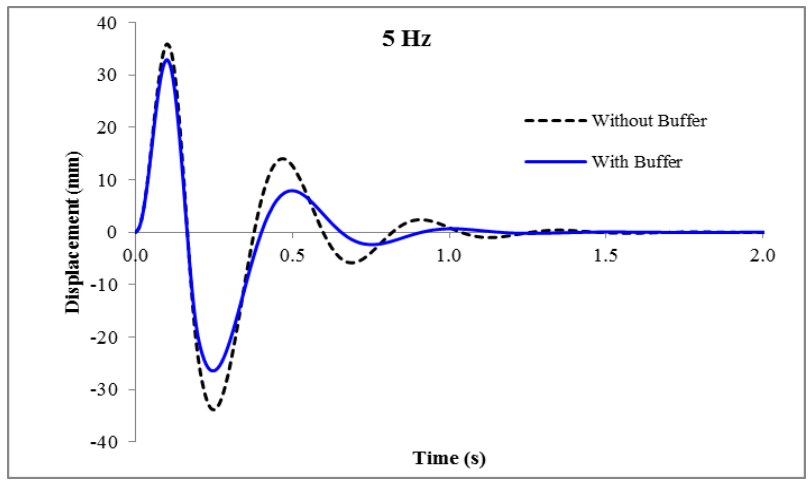

Figure 13. Displacement vs. time of the sprung mass for $5 \mathrm{~Hz}$

In input frequencies around five and ten times of natural frequency as shown in Figures 14 and 15 , it is observed that the buffer is highly effective in reduction of vibrating motion in terms of amplitude and vibration time. The cycle of oscillation for system without buffer by increasing the input frequency remains approximately constant and it is about three cycles for 10 and $20 \mathrm{~Hz}$ frequencies. Also, in system with buffer the cycles of oscillation are about two cycles. The amplitude of vibration is reduced by increasing input frequency from 10 to $20 \mathrm{~Hz}$.

Figure 16 shows the amplitude ratio of the sprung mass respect to the input excitation frequency for systems with and without buffer. The amplitude ratio is the free vibration (second cycle) amplitude per amplitude of the input 
excitation for each frequency. It is observed that in frequencies under the $1 \mathrm{~Hz}$ the buffer is not effective on the reducing the vibration amplitude. On vibration with $2 \mathrm{~Hz}$ and over frequencies the elastomeric buffer is so effective in damping the vibration.

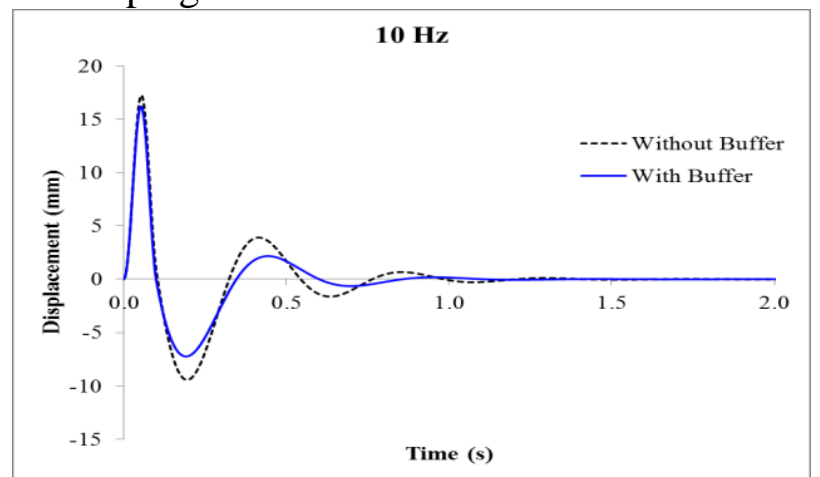

Figure 14. Displacement vs. time of the sprung mass for $10 \mathrm{~Hz}$

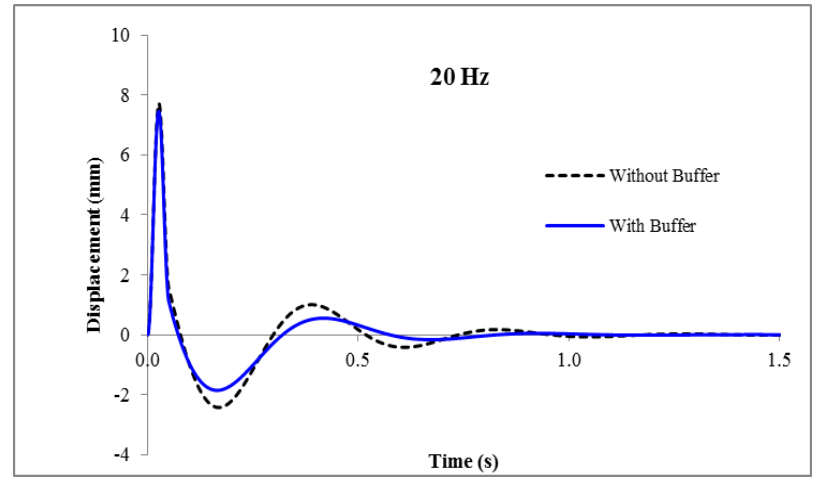

Figure 15. Displacement vs. time of the sprung mass for $20 \mathrm{~Hz}$

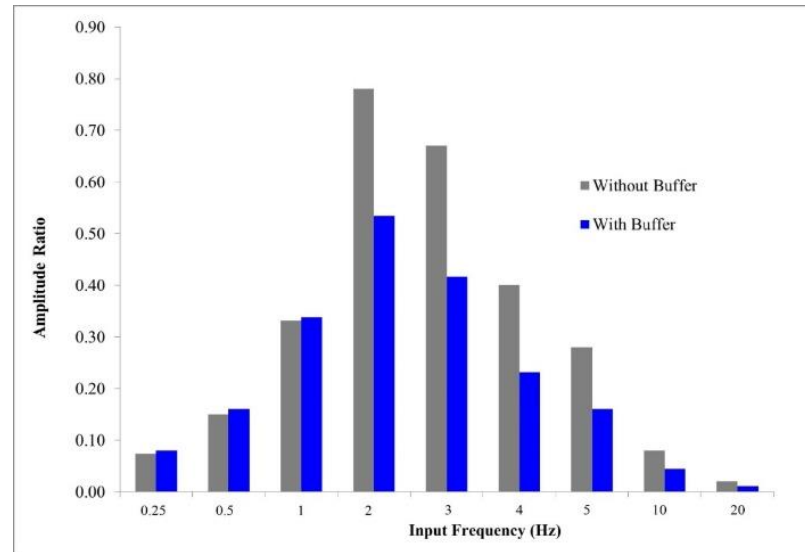

Figure 16. Amplitude ratio respect to input frequency $(\mathrm{Hz})$

In order to assess the effectiveness of buffer on reducing vibration amplitude, effectiveness percentage values were calculated as presented graphically in Figure 17. To calculate this parameter, data illustrated in Fig 16 was used. The ratios of second cycle amplitude to input excitation amplitude were obtained for each oscillation motion. Then, the ratio of obtained data for system without buffer was subtracted from data for system with buffer. Finally, the percentage of subtraction respect to the amplitude of system without buffer was obtained. The obtained results indicated that in input frequencies under the damped natural frequency of system, the buffer was not effective. In frequencies near and over the natural frequencies the buffer reduced the vibration amplitude in free oscillation between $30 \%$ and $45 \%$ and the effectiveness was improved in frequencies between 2 to $5 \mathrm{~Hz}$. In frequencies ranging 10 to $20 \mathrm{~Hz}$, the effectiveness percentage remained constant. With attention to that viscoelastic material damping properties are highly sensitive to loading frequency and there is an optimal frequency range where loss factor peaks. it is important to consider the application frequency range of buffer in design stage.

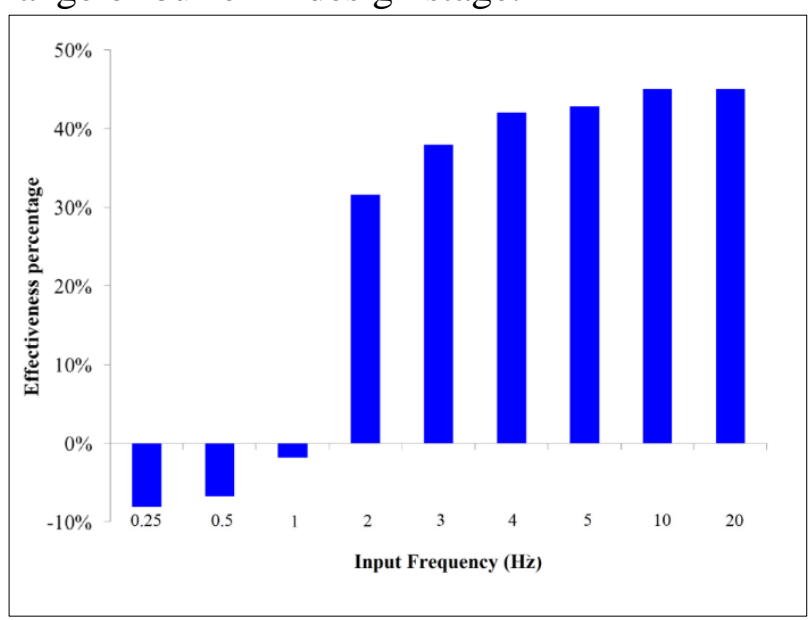

Figure 17. Effectiveness percentage respect to input frequency $(\mathrm{Hz})$

\section{Conclusion}

In this study, it was aimed to examine the performance of elastomeric buffer embedded on the coil spring of an automobile suspension system. To reach to this aim, explicit finite element method was employed. The finite element model was validated by comparing the numerical results with analytical ones. The comparison proved that numerical and analytical results are in good agreement. The displacement transmissibility ratio and the phase shift values were used to validate the numerical model. The numerical analysis results showed that the elastomeric buffer reduced the vibration amplitude and oscillation period in a 
wide range of input excitation frequencies. It may improve the ridding comfort of automobile and protects the suspension system from unexpected shocks while driving on a bumpy road. Also, in a system with elastomeric buffer the amplitude of vibration was decreased in comparison to system without buffer. This advances shock absorbing functionality of suspension system and extend the life of damper.

\section{References}

[1]. Lakes, R. Viscoelastic Materials. New York, United States of America, cambridge university press, (2009).

[2]. Jones, D. I. G. Handbook of Viscoelastic Vibration Damping. West Sussex, England: John Wiley and Sons, LTD, (2001).

[3]. Luo, R. K., Wang, W., Xu, Q.and X. Li, An energy dissipation approach on complete loading-unloading and dynamic impact predictions with experimental verification for rubber anti-vibration component. Polymer Testing 63 (2017) 314-322.

[4]. Ucar, H. and Basdogan, I. Dynamic characterization and modeling of rubber shock absorbers: A comprehensive case study Journal of Low Frequency Noise, Vibration and Active Control (2017) DOI: 10.1177/1461348417725954.

[5]. Vriend, N.M. and Kren, A.P. Determination of the viscoelastic properties of elastomeric materials by the dynamic indentation method. Polym Test (2004); 23: 369-375.

[6]. Lin, T.R., Farag, N.H. and Pan, J. Evaluation of frequency dependent rubber mount stiffness and damping by impact test. Appl Acoust (2005); 66: 829-844.

[7]. Haupt, P. and Sedlan, K. Viscoplasticity of elastomeric materials: experimental facts and constitutive modelling. Arch Appl Mech (2001); 71: 89-109.

[8]. Busfield, J.J.C., Deeprasertkul, C. and Thomas, A.G. Effect of liquids on the dynamic properties of carbon black filled natural rubber as a function of pre-strain. Polymer (2000), 41(26). pp 9219-9225.

[9]. Pacheco J.E.L., Bavastri, C.A. and Pereira, J.T. Viscoelastic relaxation modulus characterization using prony series. Latin Am J Solids Struct (2015); 12: 420-445.
[10]. Rao, M.D. Recent applications of viscoelastic damping for noise control in automobiles and commercial airplanes journal of sound and vibration. 262(2003) 457-474.

[11]. Mallik, A. K., Kher, V., Puri, M. Hatwal, $\mathrm{H}$. On the modeling of non-linear elastomeric vibration isolators, Journal of Sound and Vibration. 219 (1999) 239-253.

[12]. Richards, C.M. and Singh, R. Characterization of rubber isolator nonlinearities in the context of single- and multi-degree-of-freedom experimental systems, Journal of Sound and Vibration, (2001) 247(5), 807-834.

[13]. Chandra. N. C., Hatwal, H. and Mallik, A.K. Response of Non-linear Dissipative Shock Isolators. Journal of Sound and Vibration, (1998 ), 214 (4) : 589-603.

[14]. Shaska, K., Ibrahim, R. A. and Gibson, R. F. Influence of excitation amplitude on the characteristics of nonlinear butyl rubber isolators. Nonlinear Dynamics, (2007), 47 (1-3) :83-104.

[15]. Sjoberg, M. and Kari, L. Testing of Nonlinear Interaction Effects of Sinusoidal and Noise Excitation on Rubber Isolator Stiffness. Polymer Testing, (2003), 22 (3): 343-351.

[16]. Banic, M.S., Stamenkovic, D.S., Miltenovic, V.D., Milosevi, M.S., Miltenovi, A.V., Djeki, P.S., Rackov, M.J. Prediction of Heat Generation in Rubber or Rubber-metal Springs. Therm. Sci. (2012), 16, 527-539.

[17]. Huang, B. W., Tseng, J. G. and Ko, Y.L. Stress and vibration of a viscoelastic damping isolator under impact loading. Journal of Vibro engineering, (2014)vol. 16, no. 5, pp. 2355-2362.

[18]. Johnson, A.R.; Chen, T.K. Approximating Thermo-viscoelastic Heating of Largely Strained Solid Rubber Components. Comput. Methods Appl. Mech. Engrg. (2005), 194, 313-325.

[19]. Luo, R.K. Gabbitas, B.L. and Brickle, B.V. Fatigue design in railway vehicle bogies based on dynamic simulation Veh. Syst. Dyn., 25 (1996), pp. 438-449.

[20]. Luo, R.K., Gabbitas, B.L. and Brickle B.V. Fatigue life evaluation of a railway vehicle bogie using an integrated dynamic 
simulation J. Rail Rapid Transit, 208 (1994), pp. 123-132.

[21]. Grassie S.L. Resilient railpads their dynamic behaviour in the laboratory and on track Proc. Inst. Mech. Eng., 203 (2007), pp. 25-32.

[22]. K. Reza Kashyzadeh, M. J. OstadAhmad-Ghorabi, A. Arghavan, 'Investigating the Effect of Road Roughness on Automotive Component', Engineering Failure Analysis, (2014), Vol. 41.

[23]. K. Reza Kashyzadeh, M. J. OstadAhmad-Ghorabi, A. Arghavan, 'Study Effects of Vehicle Velocity on A Road Surface Roughness Simulation', Applied Mechanics and Materials, (2013), Vol. 372.

[24]. K. Reza Kashyzadeh, A. Arghavan, M. J. Ostad-Ahmad-Ghorabi, 'Fatigue Life Prediction of Suspension Automotive under Random Vibration Based Road Roughness', Mediterranean Journal of Modeling and Simulation, (2015) Vol. 4, Issue.1.

[25]. http://www.buykorea.org/productdetails/Power-cushion-buffer--54339.html (accessed 10 November 2017).

[26]. ABAQUS User's Manual Ver. 6.10 Dausault Systems Inc., 2010.

[27]. Nandi, B., Dalrymple, T., Yao, J. and Lapzyk, I. Importance of capturing nonlinear viscoelastic material behavior in tire rolling simulations, (2014) meeting of the tire society. 